\title{
¿Synthesis of Vortex Rossby Waves. Part III: Rossby Waveguides, Vortex Motion, and the Analogy with Midlatitude Cyclones
}

\author{
ISRAEL GONZALEZ III ${ }^{\mathrm{a}}$ AND H. E. WILlOUGHBY ${ }^{\mathrm{b}}$ \\ ${ }^{a}$ National Weather Service WFO, Tallahassee, Florida \\ ${ }^{\mathrm{b}}$ Department of Earth and Environment, Florida International University, Miami, Florida
}

(Manuscript received 30 June 2020, in final form 29 March 2021)

\begin{abstract}
Vortex Rossby waves (VRWs) affect tropical cyclones' (TCs') motion, structure, and intensity. They propagate within annular waveguides defined by a passband between $\Omega_{1 \mathrm{D}}$, the Doppler-shifted frequency of a onedimensional VRW, and zero. Wavenumber-1 VRWs cause TC motion directly and have wider waveguides than wavenumbers $\geq 2$. VRWs forced with fixed rotation frequency propagate away from the forcing. Initially outwardpropagating waves are Doppler shifted to zero at a critical radius, where they are absorbed. Initially inward-propagating waves are Doppler shifted to $\Omega_{1 \mathrm{D}}$, reflect from a turning point, propagate outward, and are ultimately absorbed at the critical radius. Between the forcing and the turning radii, the VRWs have standing-wave structure; outward from the forcing they are trailing spirals. They carry angular momentum fluxes that act to accelerate the mean flow at the forcing radius and decelerate it at the critical radius. Mean-flow vorticity monopoles are inconsistent with Stokes's theorem on a spherical Earth, because a contour enclosing the monopole's antipode would have nonzero circulation but would enclose zero vorticity. The Rossby waveguide paradigm also fits synoptic-scale Rossby waves in a meridionally sheared zonal flow. These waves propagate within a waveguide confined between a poleward turning latitude and an equatorward critical latitude. Forced waves are comma-shaped gyres that resemble observed frontal cyclones, with trailing filaments equatorward of the forcing latitude and standing waves poleward. Even neutral forced Rossby waves converge westerly momentum at the latitude of forcing.
\end{abstract}

KEYWORDS: Tropics; Hurricanes; Rainbands; Rossby waves

\section{Introduction}

Tropical cyclones (TCs) are a worldwide threat to life and property. Their impacts are increasing in step with growing coastal populations, increasing development, and arguably climate change (e.g., Knutson et al. 2010). TC structure and track can dominate local impacts. Minor track deviations can lead to significant differences in storm surge. Outer spiral rainbands are often destructive even though they are well removed from the intense vortex core. Here we explore inner-core dynamics that influence motion and rainband configuration.

A TC's primary circulation swirls cyclonically around its low pressure center in approximate gradient balance. Superimposed upon it are frictionally and diabatically forced secondary circulations that act to spin up and maintain the vortex. These flows converge moist air in contact with the ocean surface, rise in the eyewall to feed intense convection, and diverge near tropopause level. Convective latent heat release in the eyewall sustains the warm core. In radar and satellite imagery, the eyewall is surrounded by curved rainbands (Fig. 1). The objective here is insight into the dynamics of the bands themselves and their interactions with the primary and secondary circulations.

¿ Denotes content that is immediately available upon publication as open access.

Corresponding author: Hugh E. Willoughby, willough@fiu.edu
In a real, bounded hurricane, the radial profile of relative vorticity $\zeta$ is strongly cyclonic in the vortex core and surrounded by enough anticyclonic $\zeta$ that the circulation becomes zero at some finite radius. Many idealized studies are inconsistent with Stokes's theorem on a sphere because they are based upon mean-flow vorticity monopoles. Here we use an idealized barotropic nondivergent (BND) model to advance physical understanding of linear vortex Rossby waves (VRWs) propagating upon the radial gradient of axially symmetric $\zeta$ (e.g., Montgomery and Kallenbach 1997, hereafter MK97). VRWs propagate within annular waveguides defined by zero Doppler-shifted frequency and $\Omega_{1 \mathrm{D}}$, the frequency of a onedimensional wave with the same azimuthal wavenumber (Cotto et al. 2015, hereafter Part I; Gonzalez et al. 2015, hereafter Part II). A realistic, bounded vortex has an inner waveguide where the waves' intrinsic propagation is upstream, more slowly than the mean flow, and an outer waveguide where their intrinsic propagation is downstream, faster than the mean flow. Vorticity monopoles have only the downstream-propagating waveguide. This study addresses VRW's roles in TC motion, structure, and intensity change. In addition, the same modeling approach is reapplied to middle latitude Rossby waves to confirm and extend Macdonald's (1968) original analogy with synoptic-scale cyclones.

To a first approximation, TC motion can be modeled as advection by the large-scale steering flow. Absolute vorticity is conserved in frictionless, nondivergent flow. On a spherical Earth, the $\beta$ effect, where $\beta$ is the meridional gradient of the Coriolis parameter, contributes to TC motion (e.g., Holland 1983; Chan and Williams 1987). Advection of $\beta$ by 


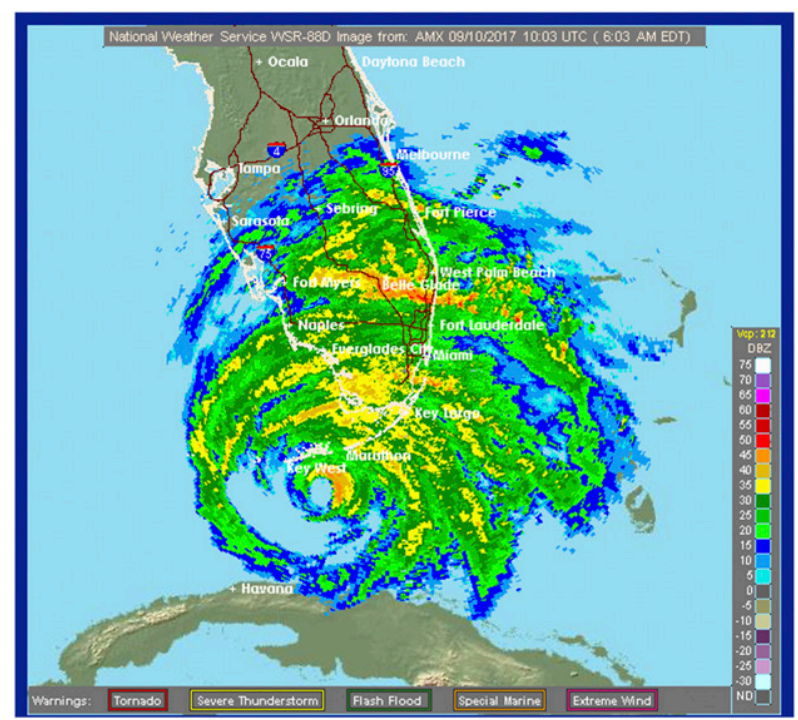

FIG. 1. Miami radar image of Hurricane Irma at 1003 UTC 10 Oct 2017, showing the heaviest precipitation in the eyewall and outer rainbands.

equatorward mean flow on the west side of the symmetric vortex and poleward mean flow on the east side combine to force a streamfunction dipole such that the flow between its gyres advects the mean vortex poleward and westward. As shown in Part II, wave-wave interactions among induced VRWs are essential to constraining the $\beta$ drift to a reasonable $1-2 \mathrm{~m} \mathrm{~s}^{-1}$.

In a BND model, rotating vorticity source-sink dipoles induce motion by forcing flow across the center (Willoughby 1992). This "convective" effect works only for wavenumber 1 because the kinematics of wavenumbers $\geq 2$ require both the streamfunction and its gradient to be zero in the neighborhood of the vortex center. Convective asymmetries, such as spiral rainbands, are more complicated than the static $\beta$ gyres because the cells rotate around the center to cause trochoidal motions observed in nature (Nolan and Montgomery 2000, hereafter NM00; Nolan et al. 2001; Lawrence and Mayfield 1977). Wavenumber-1 asymmetries also have the widest possible Rossby waveguide for a given vortex structure and imposed rotation frequency.

Macdonald (1968) first noticed that rainbands observed by radar exhibited Rossby-wave-like characteristics. Observed spiral bands tilted outward from the axis of mean-vortex rotation and moved with the mean flow, but more slowly, much as synoptic-scale troughs do. Their inward transport of cyclonic angular momentum was analogous to the poleward flux of westerly momentum in the general circulation. These analogies inspired a formal theory on VRWs (MK97): the asymmetric balance $(\mathrm{AB})$ model based upon the assumption that square of VRW's Doppler-shifted frequency was much less than the square of local inertia frequency. The AB model was governed by the linearized asymmetric vorticity equation, (1), for a simple vorticity monopole on an $f$ plane in stationary cylindrical coordinates:

$$
\left(\frac{\partial}{\partial t}+\frac{v_{0}}{r} \frac{\partial}{\partial \lambda}\right) \zeta^{\prime}-\frac{\partial \psi^{\prime}}{r \partial \lambda} \frac{\partial \zeta_{0}}{\partial r}=0
$$

Here $t$ is time; $v_{0}$ is the axially symmetric mean tangential velocity; $r$ is radius; $\lambda$ is azimuth; $\zeta^{\prime}$ and $\psi^{\prime}$ represent the perturbation vorticity and streamfunction, respectively; and $\zeta_{0}$ is the axially symmetric absolute vorticity. Outward-propagating VRW packets became nearly stationary at a finite, outer stagnation radius as their Doppler-shifted frequencies approached zero and their radial wavenumbers became large. Existence of the stagnation (or critical) radius highlighted the dependence of VRW propagation upon the mean radial vorticity gradient. MK97 hypothesized that forced VRWs transported energy from one radial band to another.

AB was tailored to represent VRWs but not inertia-gravity waves. Although, MK97 speculated that previous shallowwater, primitive equation models mistook VRWs for inertia gravity waves, the faster intrinsic propagation of gravity waves and their ability to radiate into the storm environment support a clear distinction. By analogy with nondivergent synopticscale Rossby waves, MK97 derived a VRW dispersion relation, (2), which showed that VRWs propagate against the cyclonic angular velocity in the vortex core when the mean vorticity gradient is negative:

$$
\Omega(r)=\omega-\frac{n v_{0}}{r}=\frac{n}{r} \frac{\partial \zeta_{0} / \partial r}{\left(k^{2}+\frac{n^{2}}{r^{2}}\right)} .
$$

Here, $\Omega(r)$ is the Doppler-shifted frequency, $\omega$ is the wave train's rotation frequency relative to the ground, $n$ is the tangential wavenumber, and $k$ is the local [in the WentzelKramers-Brillouin (WKB) sense] radial wavenumber. The dispersion relation describes the VRW's propagation dynamics. Both $\omega$ and $n$ are fixed for a given wave train. Since $\partial \zeta_{0} / \partial r<$ 0 in the inner waveguide, the waves rotate more slowly than the wind within a frequency passband $\left(0 \geq \Omega \geq \Omega_{1 \mathrm{D}}=\left(\partial \zeta_{0} / \partial r\right)(n / r)^{-1}\right.$. When the $\Omega$ approaches zero at the critical (stagnation) radius, $k$ becomes large; $k$ approaches zero when $\Omega \rightarrow \Omega_{1 \mathrm{D}}$. Further notable results from $\mathrm{AB}$ theory included linear phase and group velocities, identification of VRWs with at least some observed spiral bands, and recognition of VRWs potential role in TC intensification.

Subsequent research confirmed that angular momentum transport by forced VRWs may cause eyewall contraction and mean-flow intensification (Chen and Yau 2001; Möller and Shapiro 2002; Chen et al. 2003). The first of these studies identified prograde VRWs that propagate faster than the tangential winds where $\partial \zeta_{0} / \partial r>0$ on the inward sides of potential vorticity rings and carry eddy vorticity fluxes toward the eyewall. Chen et al. (2003) specifically noted acceleration of the mean tangential wind in the lower and middle troposphere and deceleration aloft. At least two studies (Wang 2002; Chen and Yau 2003) argued that wave-mean flow interactions actually preventing intensification because the VRWs accelerated tangential winds in the eyewall directly leading to axially symmetric outflow and deceleration at the RMW.

Theoretical studies provided insight into VRW dynamics, but existence of VRWs in real TCs remained uncertain. 
Aircraft and radar observations in eastern Pacific Hurricanes Jimena of 1991 and Olivia of 1994 (Reasor et al. 2000; Black et al. 2002) revealed low-wavenumber, cyclonically rotating eyewall perturbations, vorticity asymmetries, and rainbands with VRW-like properties (see Muramatsu 1986; Kuo et al. 1999). In Hurricane Olivia, spiral bands of vorticity were found in regions where wavenumber-2 vorticity asymmetries appeared to be symmetrizing. Asymmetries exhibited rotation rates slower than the mean flow's angular velocity near deep, intense convection at the RMW. Hurricanes Jimena and Olivia were sheared storms with periodic convection that exhibited radar echoes and updrafts forming in the downshear quadrant. The reflectivity asymmetries were advected around the eye at $60 \%-80 \%$ of the swirling wind, consistent with convectively excited VRW propagation. Numerical simulations and theoretical studies of these features generally validated the observational findings (Chen and Yau 2001; Wang 2002; MK97).

Despite the foregoing advances in understanding of VRW dynamics, the waves' effects on TC motion, and the waveguides within which they propagate have received limited attention. Part I built upon previous work with frequencydomain simulations of episodically forced, BND VRWs with wavenumbers $\geq 2$ in the inner waveguide of bounded vortices. Part II simulated wavenumber 1 VRWs in time domain on a $\beta$ plane to demonstrate that wave-wave interaction was essential to reasonable poleward and westward $\beta$-drift speeds. The primary motivation here is to extend Part I's insights to wavenumber 1 , which can force TC motion and has the widest possible waveguide for given $\omega$. The model focuses on convectively forced wavenumber 1 , as in Part I, but uses the time-domain approach of Part II. Section 2 describes formulation of the BND model in translating cylindrical coordinates. Section 3 applies it to the convectively forced wavenumber 1 at different frequencies. Section 4 revisits the $\beta$-plane problem and section 5 examines vorticity monopoles on a spherical Earth. Section 6 connects VRWs back to Macdonald's (1968) original analogy with midlatitude cyclones, and section 7 summarizes the results and draws conclusions.

\section{Formulation}

A BND, vortex-following, semispectral model set in a still environment on an $f$ plane is the simplest formulation able to simulate vortex motion and $2 \mathrm{D}$ convectively forced wavenumber-1 VRWs. The model, written in MATLAB, accepts as inputs the mean vortex and wave forcing, and marches the vorticity equation forward in time in translating cylindrical coordinates (Fig. 2). After each time step, the vorticity is inverted to obtain the streamfunction. The moving vortex center corresponds to the coordinate's origin. It is tracked by minimizing the $\alpha$-gyre apparent asymmetry due to mislocation of the mean-vortex center (Willoughby 1992).

The mean vortex structure derives from the Wood and White (2010) formulation (Fig. 3). The radial profile of the cyclostrophic wind inside the RMW obeys U-shaped power law with an exponent of 1.1; outside the RMW it obeys an inverse power law with an exponent of -0.5 (e.g., Riehl 1963);

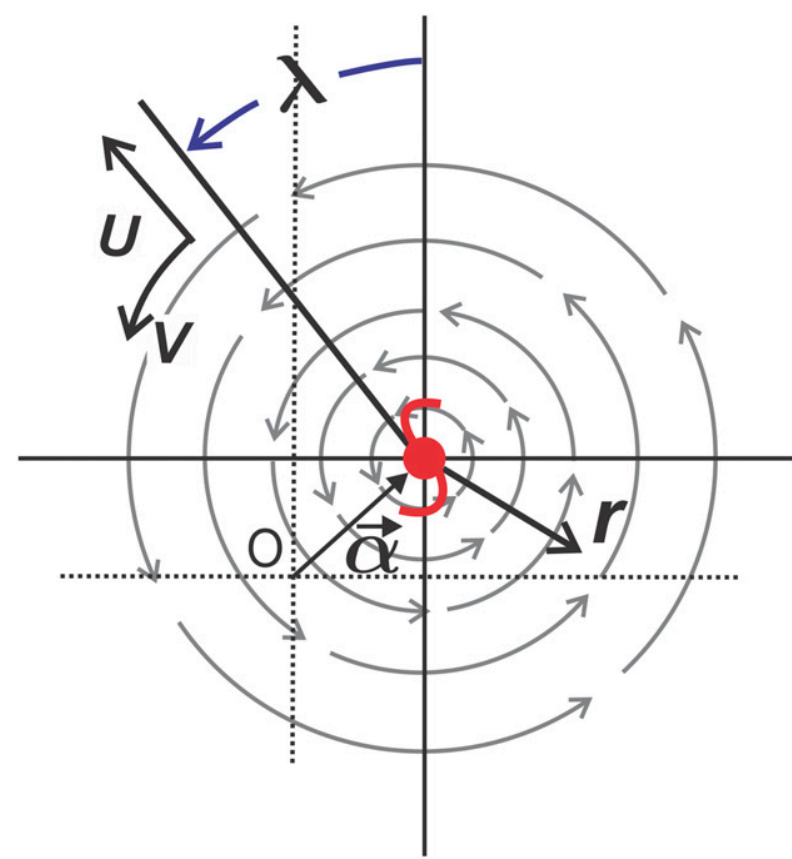

FIG. 2. Vortex-following cylindrical coordinates used in the present analysis (Part II). Here $O$ is the origin, the hurricane symbol marks the vortex center, $\boldsymbol{\alpha}$ is the centering error, and the other variables are as described in the text.

and it has a smooth, though reasonably sharp, transition (Wood-White $\lambda=0.25$ ) across the RMW. The RMW is $25 \mathrm{~km}$ and maximum cyclostrophic is $50 \mathrm{~m} \mathrm{~s}^{-1}$. The profile used is the gradient wind derived from the foregoing cyclostrophic profile at $20^{\circ}$ latitude. This formulation is more logically consistent because the cyclostrophic profile has a well-defined pressurewind relation and the gradient wind's circulation goes asymptotically to zero as $r$ becomes large. It is thus an asymptotically bounded vortex rather than an unbounded vortex.

Vorticity is the prognostic variable in the present BND model. The axially symmetric mean-flow relative vorticity is strongly cyclonic in the vortex core, weakens with increasing radius, and reverses sign at the periphery before increasing asymptotically to zero. The mean radial vorticity gradient, $\partial \zeta_{0} / \partial r$, is generally negative in the core, but can be positive in a narrow interval within the eye. The derivation of the vorticity equation begins with the wavenumber- 1 radial and tangential momentum equations, (3) and (4), in translating cylindrical coordinates (e.g., Willoughby 1992):

$$
\begin{gathered}
\frac{\partial u}{\partial t}+\frac{v_{0}}{r} \frac{\partial u}{\partial \lambda}-\xi_{0}\left(v-c_{\lambda}\right)+\frac{\partial \phi}{\partial r}=F_{r}, \\
\frac{\partial v}{\partial t}+\frac{v_{0}}{r} \frac{\partial v}{\partial \lambda}+\zeta_{0}\left(u-c_{r}\right)+\frac{1}{r} \frac{\partial \phi}{\partial \lambda}=F_{\lambda} .
\end{gathered}
$$

Here $u$ is radial velocity (positive outward), $v$ is tangential velocity (positive cyclonically), $\phi$ is geopotential, $\lambda$ is azimuth (cyclonically from north), $r$ is radius, $c_{r}$ and $c_{\lambda}$ are the radial and tangential components of the coordinates translation, $F_{r}$ and $F_{\lambda}$ are radial and tangential momentum forcing, 


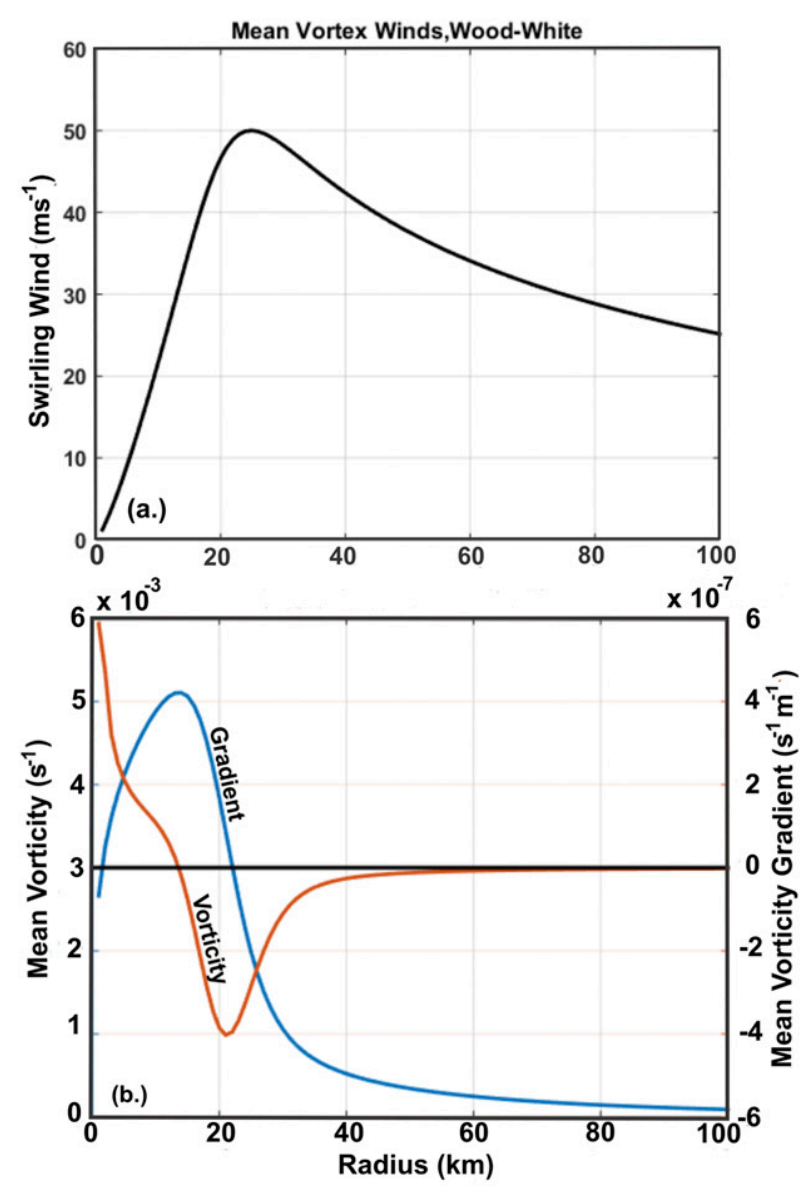

FIG. 3. (a.) Mean tangential wind profile and (b) vorticity (red) and vorticity gradient (blue).

and $\xi_{0}=2 v_{0} / r+f$ is the inertia parameter. The forcing is derived from the gradient of a scalar $A$ such that it is nondivergent. The complex motion is $C=c_{x}+i c_{y}$, where $c_{x}$ and $c_{y}$ are its zonal and meridional components, so that $c_{r}=$ $\operatorname{Re}\left(-i C e^{-i \lambda}\right)$ and $c_{\lambda}=\operatorname{Re}\left(C e^{-i \lambda}\right)$. Cross differentiation and subtraction eliminates $\phi$ to yield the nondivergent wavenumber-1 vorticity equation:

$$
\begin{gathered}
\left(\frac{\partial}{\partial t}+\frac{v_{0}}{r} \frac{\partial}{\partial \lambda}\right)\left(\frac{\partial v}{\partial r}+\frac{v}{r}-\frac{1}{r} \frac{\partial u}{\partial \lambda}\right)+\left(u-c_{r}\right) \frac{\partial \zeta_{0}}{\partial r} \\
=\frac{\partial^{2} A}{\partial r^{2}}+\frac{1}{r} \frac{\partial A}{\partial r}+\frac{1}{r^{2}} \frac{\partial^{2} A}{\partial \lambda^{2}} \equiv Q
\end{gathered}
$$

The imposed forcing $Q$ is the Laplacian of $A$. In the frequency domain, the wavenumber- 1 solution is the real part of the product of the complex amplitude of the streamfunction, $\Psi(r, t)$, with an imaginary exponential in azimuth, $\psi=\operatorname{Re}\left\{\Psi(r, t) e^{i(\omega t-\lambda)}\right\}$ such that $v=\partial \psi / \partial r$ and $u=-r^{-1} \partial \psi / \partial \lambda$ :

$$
\Omega\left(\frac{\partial^{2} \Psi}{\partial r^{2}}+\frac{1}{r} \frac{\partial \Psi}{\partial r}-\frac{\Psi}{r^{2}}\right)-\left(\frac{\Psi}{r}-C\right) \frac{\partial \zeta_{0}}{\partial r}=Q .
$$

The amplitude of perturbation vorticity is

$$
\Xi=\frac{\partial^{2} \Psi}{\partial r^{2}}+\frac{1}{r} \frac{\partial \Psi}{\partial r}-\frac{\Psi}{r^{2}}
$$

Rearranging the terms in (6) yields the vorticity tendency equation which is expanded with finite differences to march $\zeta$ forward in time:

$$
\frac{\partial \zeta}{\partial t}=-\frac{v_{0}}{r} \frac{\partial \zeta}{\partial \lambda}-\left(u-c_{r}\right) \frac{\partial \zeta_{0}}{\partial r}+Q
$$

$\Psi$ is computed by inversion of (7) with the Lindzen and Kuo (1969) algorithm, and eddy fluxes of geopotential and angular momentum and their convergences are calculated. The boundary conditions imposed are $\partial \psi / \partial r=0$ at the center and the periphery. As a practical matter the solutions are insensitive to them since the evanescent tails have enough distance to decay between the turning or critical radius and the boundaries.

The dispersion relation, [(2), offers insight into VRW propagation. If $\Psi(r, t)$ is represented with zero-order Hankel functions, (6) becomes

$$
\Omega=\omega-\frac{v_{0}}{r}=\frac{r^{-1} \partial \zeta_{0} / \partial r}{k_{r}^{2}+r^{-2}}
$$

where $k_{r}$ is the local radial wavenumber. As shown above, radially long VRWs approach the Rossby cutoff frequency, $\Omega \rightarrow \Omega_{1 \mathrm{D}}=r \partial \zeta_{0} / \partial r$, at the inner boundary of the waveguide. Short waves' Doppler-shifted frequencies approach zero, $\Omega \rightarrow$ 0 at the outer critical radius. Radial phase and group velocities, (10), are obtained as in MK97:

$$
C_{p r}=\frac{1}{k_{r}}\left(\frac{v_{0}}{r}+\frac{r^{-1} \partial \zeta_{0} / \partial r}{k_{r}^{2}+r^{-2}}\right), \quad C_{g r}=-\frac{2 k_{r} r^{-1} \partial \zeta_{0} / \partial r}{\left(k_{r}^{2}+r^{-2}\right)^{2}}
$$

VRW's propagation characteristics are consistent with that of midlatitude Rossby waves. The radial wavenumber determines the sense of propagation. If $k_{r}$ is positive, phase propagates outward and energy propagates inward; if it is negative phase propagates inward and energy propagates outward. This situation contrasts with tangential propagation in the inner waveguide, where phase always propagates upstream relative to the mean flow, and energy may propagate upstream or downstream dependent upon whether $k_{r}$ is larger or smaller than $r^{-1}$.

\section{Forced wavenumber 1}

This section examines wavenumber-1 VRWs forced at radius $r_{q}$, specified to be the RMW, with rotation frequencies: $0.25 \omega_{q}, 0.50 \omega_{q}, 0.75 \omega_{q}$, and $\omega_{q}$, where $\omega_{q}=v_{0}\left(r_{q}\right) / r_{q}$. The analysis presents the solution expressed in terms of both $\zeta$, to highlight propagation within the waveguide, and $\psi$, to illustrate induced, vortex-scale flow that extends beyond the waveguide. It also depicts mean-vortex track and eddy fluxes of angular momentum and geopotential to understand interaction with the mean flow. The rotation frequency $\omega$ determines the waveguide geometry and where flux convergences and divergences occur. Comparison of wavenumber-1 results with Part I's higher-wavenumber VRW are informative. 


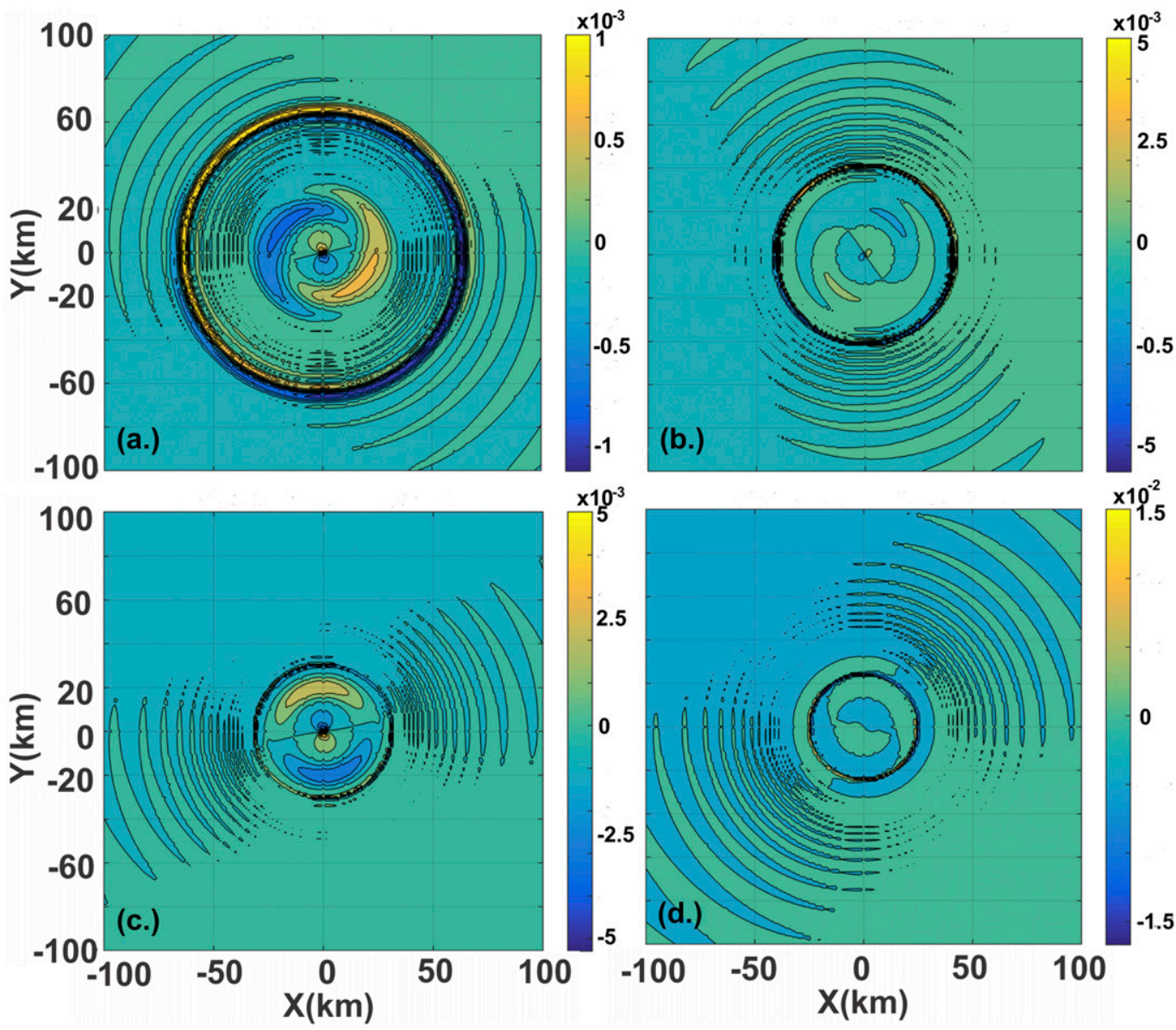

FIG. 4. Contour plots of wavenumber-1 perturbation vorticity after $24 \mathrm{~h}$ for specified forcing orbital periods: (a) 3.5, (b) 1.8 , (c) 1.2, and (d) $0.87 \mathrm{~h}$, showing symmetrized trailing spirals at the end of each simulation.

The forcing is localized in a $20-\mathrm{km}$-wide annulus centered at $r=20 \mathrm{~km}, 5 \mathrm{~km}$ inside the RMW, and is zero everywhere else (Part I). It rotates with the specified frequency $\omega$, has a bell-shaped radial structure, wavenumber-1 azimuthal structure, and is constant in time apart from the rotation. Since the forcing is continuous, only the gyres' orientation changes with time as the dipole rotates after startup transients have died out. For example, $\omega=0.25 \omega_{q}$ corresponds to a 3.5 -h orbital period $(T)$, or approximately 7 full rotations per simulated day. This aspect of the forced dipole's behavior is similar to the algebraically growing NM00 instability.

The vorticity field appears as a rotating wavenumber- 1 dipole centered at $r_{q}$, the forcing radius (Fig. 4). A smaller dipole appears near the center of the vortex, because of $\partial \zeta_{0} / \partial r>0$ near the center, as described by NM00. The perturbations symmetrize with time, as $\zeta$ becomes filamented at the critical radius, to produce an outer ring of tightly wound, interlocking spirals, and the filamentation time (Tsai et al. 2010) becomes short. Ripple-like filaments between the dipole and ring are outward-propagating VRWs. The forcing produces a weak vorticity gradient across the domain. Subtle vorticity ripples modulate the boundary between cyclonic and anticyclonic broadscale vorticity to form a low-amplitude "wake" that aligns more or less with the vortex motion.

For most frequencies, vorticity is dominated by two features: the forced dipole near the RMW and filaments in the neighborhood of the critical radius. Short, low-amplitude, waves fill the middle of the waveguide. Beyond the critical radius, the oscillations are evanescent. Higher rotation frequencies narrow the waveguide and compress the wave pattern (Figs. 4b,c). Ultimately when $\omega=\omega_{q}$, the critical radius overlaps the forcing so that the waves are radially evanescent throughout, consistent with Part I.

The corresponding streamfunctions (Fig. 5) exhibit inner dipoles of opposite sign to the forced vorticity gyres. At low frequencies (periods $=3.5$ and $1.8 \mathrm{~h}$, Figs. 5a,b) they have much larger trailing spirals that become smaller and morph into circular gyres (period $=1.2 \mathrm{~h}$, Fig. $5 \mathrm{~b}$ ) and finally to leading spirals (period $=0.87 \mathrm{~h}$, Fig. $5 \mathrm{c}$ ) as $\omega_{q}$ increases. The outer gyres/spirals arise because of the way the vorticity filaments interact. Over much of the circumference of the critical radius, equal numbers of filaments with opposite sign occupy the same azimuth. They mask each other in the inversion for 


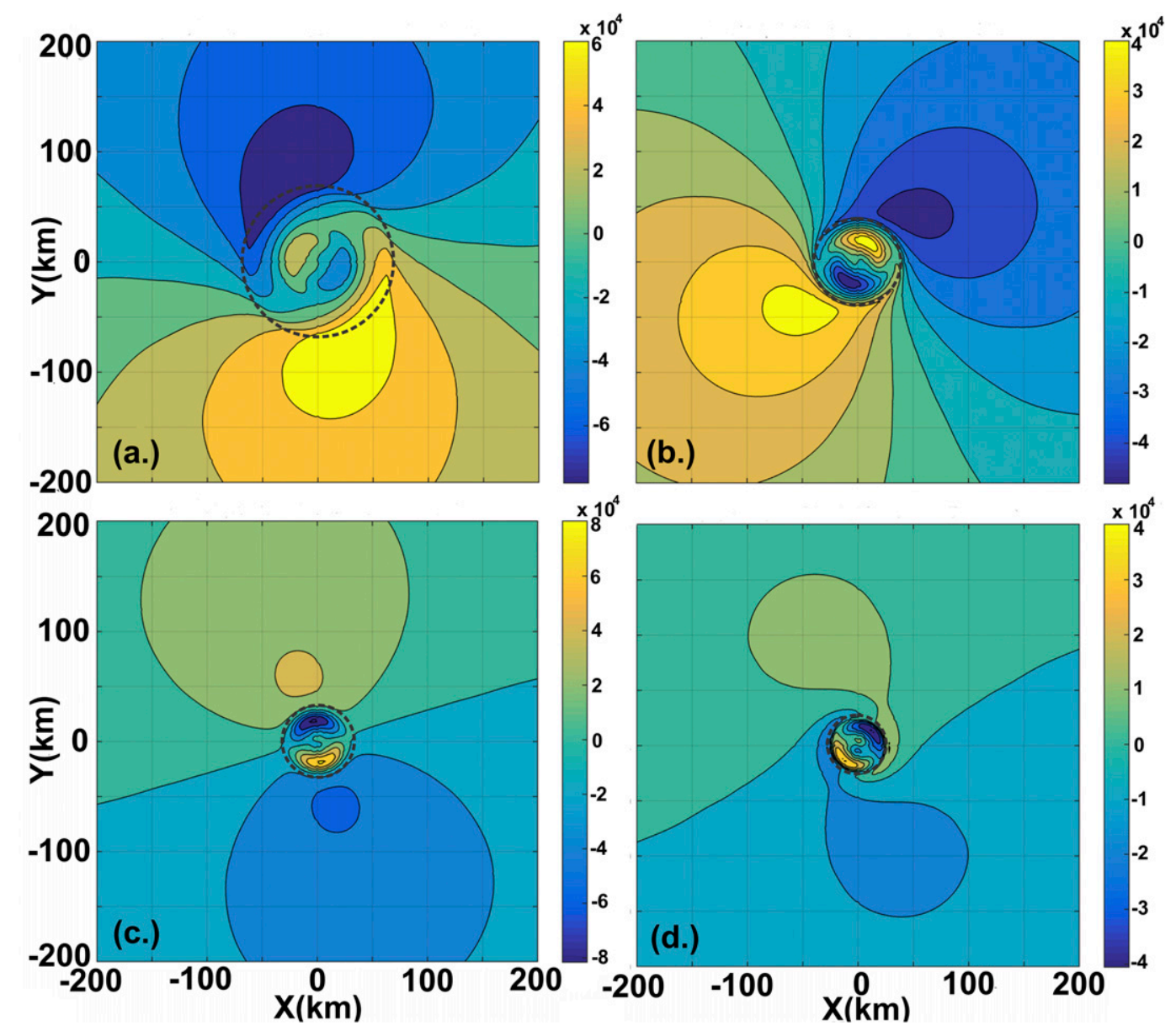

FIG. 5. Contour plots of wavenumber-1 perturbation streamfunction for the same specified duration orbital periods shown in Fig. 4. Note the change of scale. The dashed circles mark the critical radii from Fig. 4.

the streamfunction because of their small spatial scale, but when filaments of one sign or the other predominate they occupy the centers of the gyres on opposite sides of the center. There, arcs of net vorticity induce the streamfunction gyres. The resulting field causes cross-vortex flow between the gyres that advects the axially symmetric mean vorticity to induce vortex motion.

Flow across the center is different from that in Part I because the center boundary condition for wavenumbers $\geq 2$ requires both zero streamfunction and zero radial derivative across the center, whereas wavenumber 1 requires zero streamfunction, but nonzero gradient because the streamfunction changes sign across the center. The result is nonzero flow across the center that rotates with gyres to cause orbital vortex motion.

Initially the forcing causes a chaotic start-up transient before the solution stabilizes. The track then spirals cyclonically outward from the initial position and soon becomes a circular orbit at nearly constant speed (Fig. 6). Orbital speeds and radii vary with frequency from 2.42 to $3.65 \mathrm{~m} \mathrm{~s}^{-1}$ and from $\sim 1$ to $5 \mathrm{~km}$, respectively. Note that these orbital radii are significantly less than the RMW. Both motion parameters are sensitive to $\omega$.
The orbital motion arises because net asymmetric vorticity accumulates at the critical radius. It induces the outer streamfunction gyres, which support flow across the center and vortex motion. The orbital speed is the product of orbital radius with $\omega$. For a fixed frequency, increased orbital radius is the only way to increase the speed. Nonetheless, the speed generally increases incrementally with decreasing period, except when $\omega=\omega_{q}$, where the vortex has the slowest speed. By contrast, Willoughby (1992) showed the speed was fastest when the forcing rotated with mean-vortex flow's orbital period.

Vortex Rossby waves can change TC structure and intensity through eddy energy and angular momentum transports (Fig. 7). For example, NM00 noted that eddy fluxes due to their algebraic instability adjusted the wind profile inside the RMW toward solid-body rotation. In the present simulations, the energy and momentum fluxes are $\langle\phi u\rangle=$ $r \oint(\phi u) d \lambda$ and $\langle r v u\rangle=r \oint(r v u) d \lambda$, respectively. For a $3.5-\mathrm{h}$ period (Fig. 7a), the magnitudes of the acceleration and deceleration are nearly identical, so that the net result is inward redistribution of mean-flow angular momentum. In a 

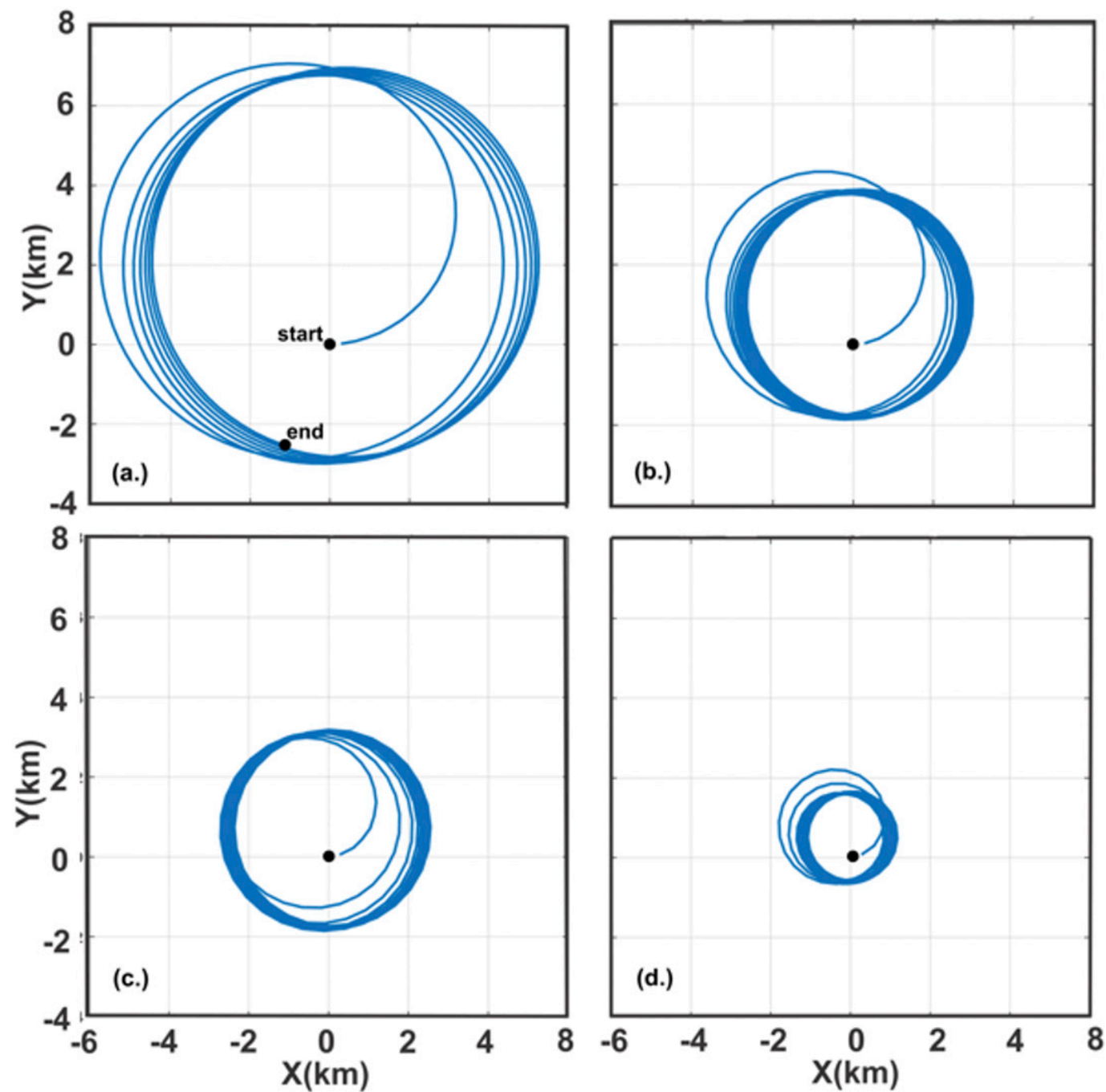

FIG. 6. Vortex tracks showing cyclonic orbits for the same duration orbital periods shown in Fig. 4.

barotropic model, these fluxes would cause the vortex spinup at the forcing locus (e.g., Gao and Zhu 2016). In a real hurricane, the momentum sink at the critical radius would force compensating inflow (Andrews and McIntyre 1976a,b; Boyd 1976) to replace the eddy-flux deficit. It is reasonable to suppose that inflow would also bring CAPE to feed convection near a developing outer wind maximum.

As shown schematically in Fig. 8, outside the forcing radius, initially outward-propagating VRWs sustain an outward energy flux and inward angular momentum flux. Inside the forcing radius, the initially inward-propagating waves are reflected from the turning radius. Since the initially inward-propagating and reflected fluxes are nearly equal and opposite, the result is essentially zero net fluxes there. Outside the forcing locus, the initially outward-propagating and reflected fluxes combine to essentially double the outward energy flux and inward angular momentum flux. The net result is strong $\langle r v u\rangle$ convergence that acts to accelerate the mean flow at the forcing, and $\langle r v u\rangle$ divergence that acts to decelerate the mean flow in the neighborhood of the critical radius. This effect would act to force localized axially symmetric convergence around the critical radius in nature or full-physics models.

In frequency space (Fig. 9a) the inner waveguide lies between the loci where $\Omega=\left(\omega-v_{0} / r\right)=0$ (blue) and $k$ is large, and $\Omega=\Omega_{1 \mathrm{D}}$ (red) and $k \rightarrow 0$, as shown previously. It is possible to contrive situations where VRWs are trapped between two turning radii (Gonzalez 2019), but that circumstance is of limited interest (Fig. 9b). Wavenumbers 2 and 3 exhibit narrower waveguides (Figs. 9c,d). VRWs obey the dispersion relation, (9), so that the wave packets propagate upstream relative to the mean flow. In the limit of large $k_{r}$, such that the waves become radially short, $\Omega \rightarrow 0$. The rotation frequency with respect to the ground, $\omega>0$, is constant. It determines how fast the wave train rotates as a whole and the width of the waveguide. Slow rotation yields wider waveguides and passbands. Critical radii for $0.25 \omega_{q} \leq \omega \leq \omega_{q}$ range between 130 and $25 \mathrm{~km}$, whereas the turning radius remains essentially fixed at $\sim 20 \mathrm{~km}$. 

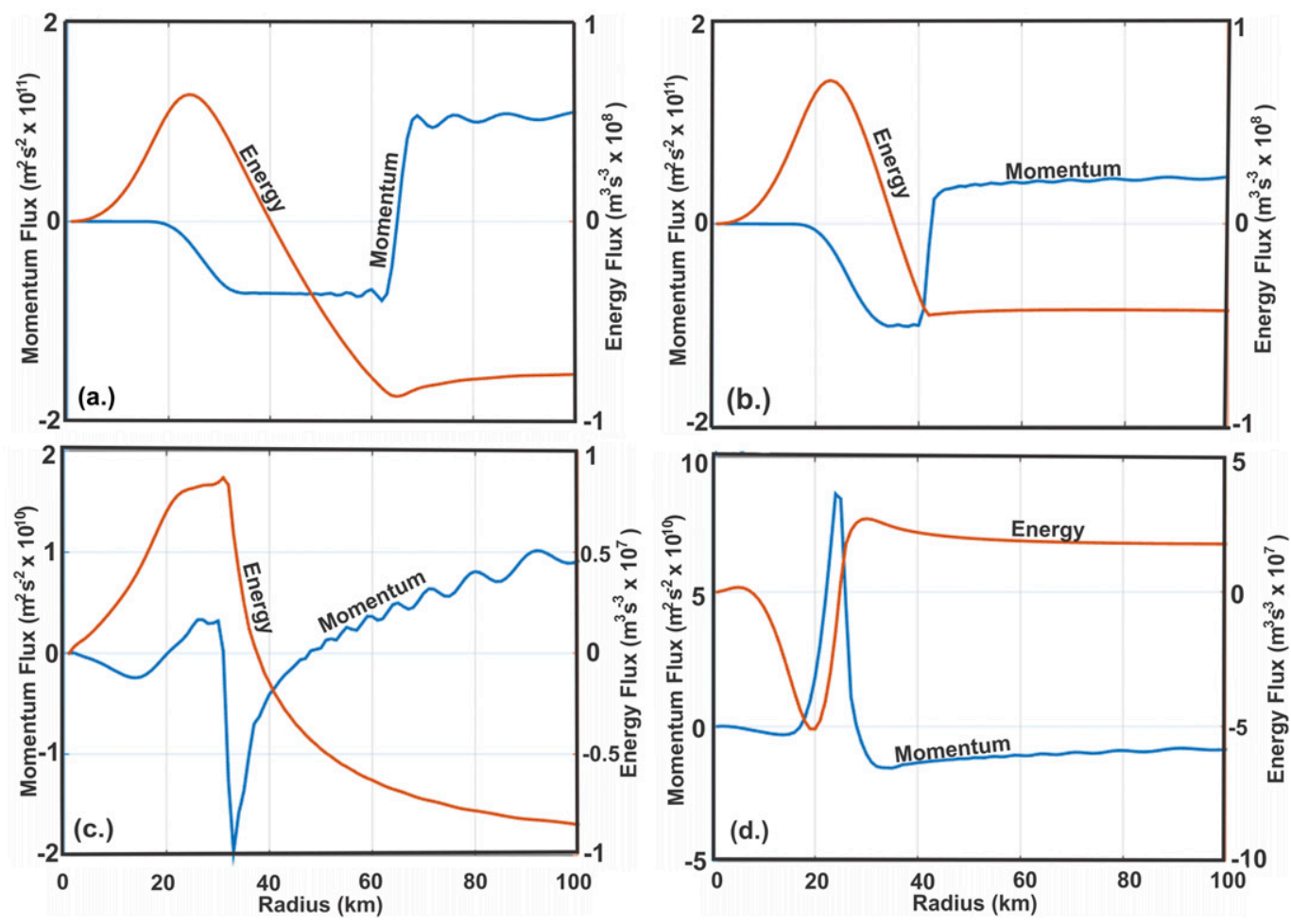

FIG. 7. Wave fluxes of angular momentum (blue) and geopotential fluxes (red) for the same orbital periods shown in Fig. 4.

\section{Beta-plane simulations}

Running the convectively forced model on a $\beta$ plane highlights the different responses to these forcings. The solutions are linear superpositions of the inner gyres forced by the mass source-sink, and outer $\beta$ gyres. The $\beta$ gyres are forced VRWs in the outer waveguides of bounded vortices where the meanflow radial vorticity gradient is weakly positive. These waves are forced at zero frequency. In the outer waveguide only lowfrequency, downstream-propagating free waves can exist. Inward-propagating free waves would be Doppler shifted to zero frequency and absorbed at the critical radius outside the vortex core. Outward-propagating free waves would be Doppler shifted to the cutoff frequency and reflected from an outer turning point before ultimately reaching the critical radius where their energy would be absorbed (Part II). This arrangement is the reverse of what happens in the inner waveguide where VRWs are confined by an inner turning point and outer critical radius. The locus of vorticity gradient sign reversal acts as the boundary between the waveguides, where VRWs propagate with different frequencies and oppositely directed azimuthal phase velocities.

In an idealized experiment where the $\beta$ effect turns off after $12 \mathrm{~h}$ while the rotating vorticity forcing dipole persists, the poleward/westward translation accelerates when $\beta$ is active and then decelerates gradually as the trochoidal motion persists through the end of the experiment after 24 simulated hours (Fig. 10a). When the mass source-sink stops at $12 \mathrm{~h}$ without turning off the $\beta$ effect, the immediate response is rapid damping of the trochoidal motion to produce smooth, slowly accelerating northwestward track (Fig. 10b). When both "convective" and $\beta$ forcing stop simultaneously at 12 simulated

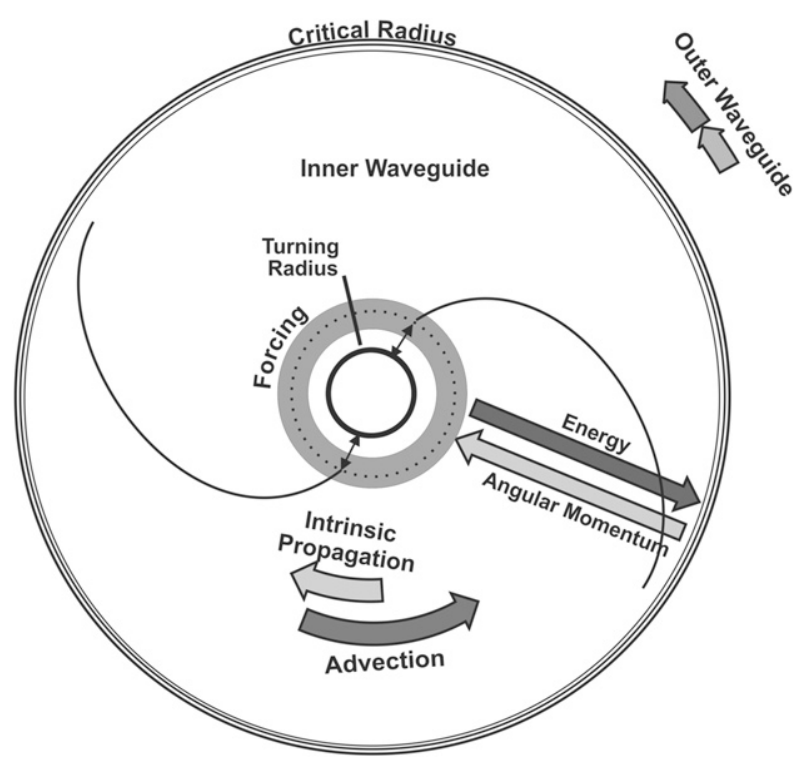

FIG. 8. Schematic diagram of VRWs propagating in the inner waveguide, illustrating standing-wave structure inward from the forcing spiral-shaped phase lines and outward from the forcing, and the senses of the eddy transports and phase propagation. 

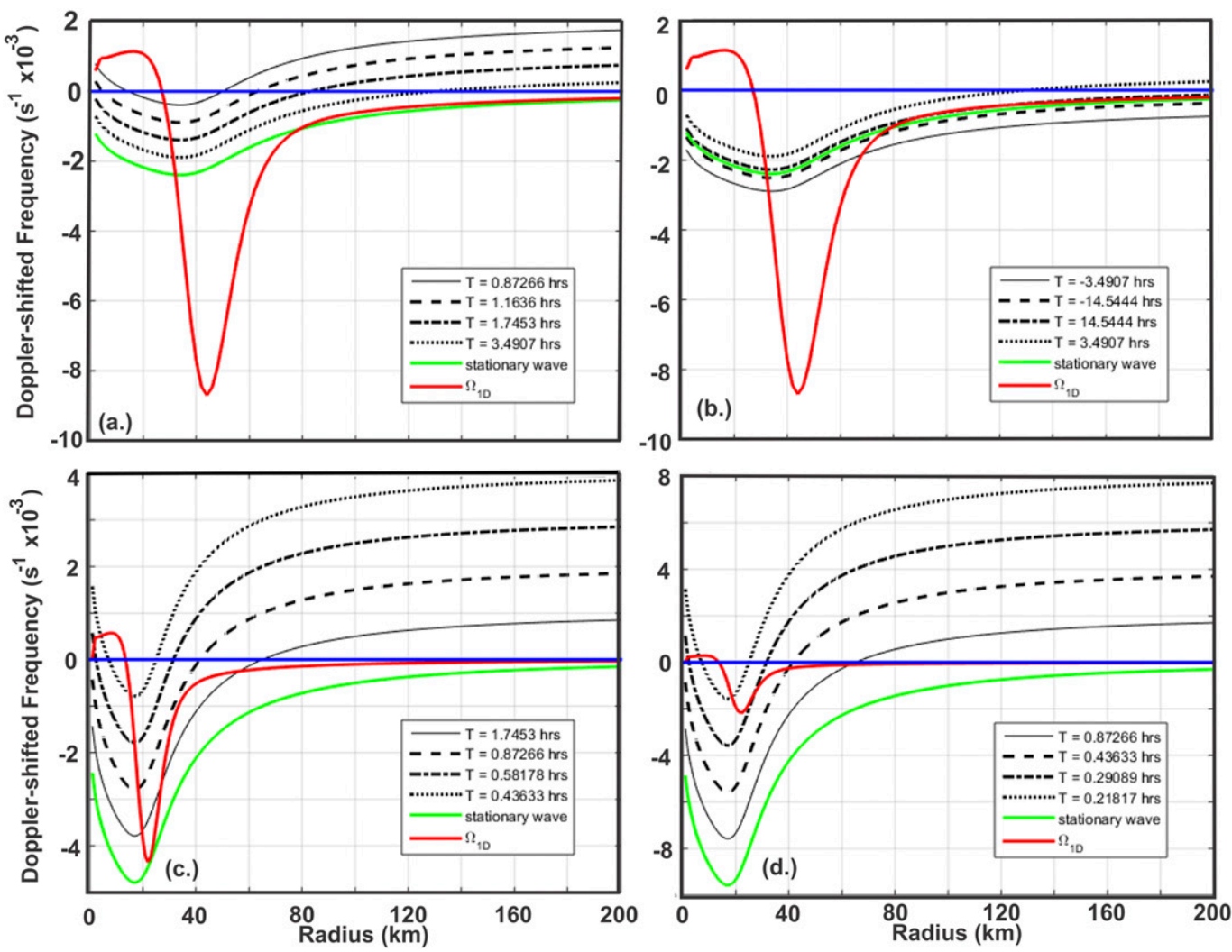

FIG. 9. (a),(b) Wavenumber-1 inner waveguide showing the frequency passbands between the cutoff frequency (red) and zero frequency (blue) representing (a) VRWs in a passband between the cutoff and zero frequency and (b) VRWs in a passband with cutoff frequencies at the inner and outer bounds of the waveguide. (c) Wavenumber- 2 and (d) wavenumber- 3 inner waveguides that are much narrower than wavenumber 1.

hours, the trochoidal motion dies out in less than a single orbit, while the $\beta$ drift decelerates to about $2 / 3$ of its speed at the transition by the simulation's end (Fig. 10c). In this case the forced $\beta$ gyres project onto a free wave with almost the same structure when the forcing stops. It persists because its group velocity is slow and spatial scale is large so that the energy loss at the outer waveguide's critical radius is gradual in contrast with the oscillatory motion which is quickly damped by filamentation at the inner critical radius. These results are superpositions of two linear solutions that do not interact dynamically. Nonetheless, they highlight the inherently differing time scales that characterize VRWs in the inner and outer waveguides. Additional sensitivity studies appear in Gonzalez (2019).

\section{Vorticity monopoles on a spherical Earth}

Unbounded mean vortices, those with nonzero circulation at large radius, have an inherent physical contradiction (Fig. 11). For a hypothetical cyclonic vorticity patch on a spherical Earth, surrounded by zero vorticity elsewhere, circulation around any contour encircling it would be equal to the patch's vorticity enclosed (e.g., Davis 1961). From the perspective of the patch's antipode, the same contour would have an anticyclonic circulation that enclosed zero vorticity. This situation contradicts Stokes's theorem. More generally, the component of the curl normal to the surface of any closed manifold must integrate to zero over the whole surface. If there were a patch of net vorticity, a singularity would arise around the antipode where the wind would approach infinity as the circulation remained constant while the contour's circumference approached zero. Thus, any physically consistent vortex must be bounded on a spherical domain.

Cyclonic vorticity near the center must be surrounded by an annulus containing an equal total amount of anticyclonic relative vorticity. This consideration requires a reversed outward radial vorticity gradient and an outer waveguide where $\partial \zeta_{0} / \partial r>0$. For any physical vortex, VRWs in the outer waveguide propagate downstream, faster than the mean swirling flow.

On the other hand, a "Rankine-like" vortex, where the swirling wind is inversely proportional to the great-circle distance from the vorticity patch, avoids this seeming contradiction. Since the circulation in that case decreases away from the vorticity patch and ultimately becomes zero as the contour length vanishes, such a vortex would, in fact, be bounded, as are vortices where the swirling flow is explicitly zero beyond some finite radius. Bounded vortices have outer waveguides where VRWs propagate downstream because the negative mean-flow 

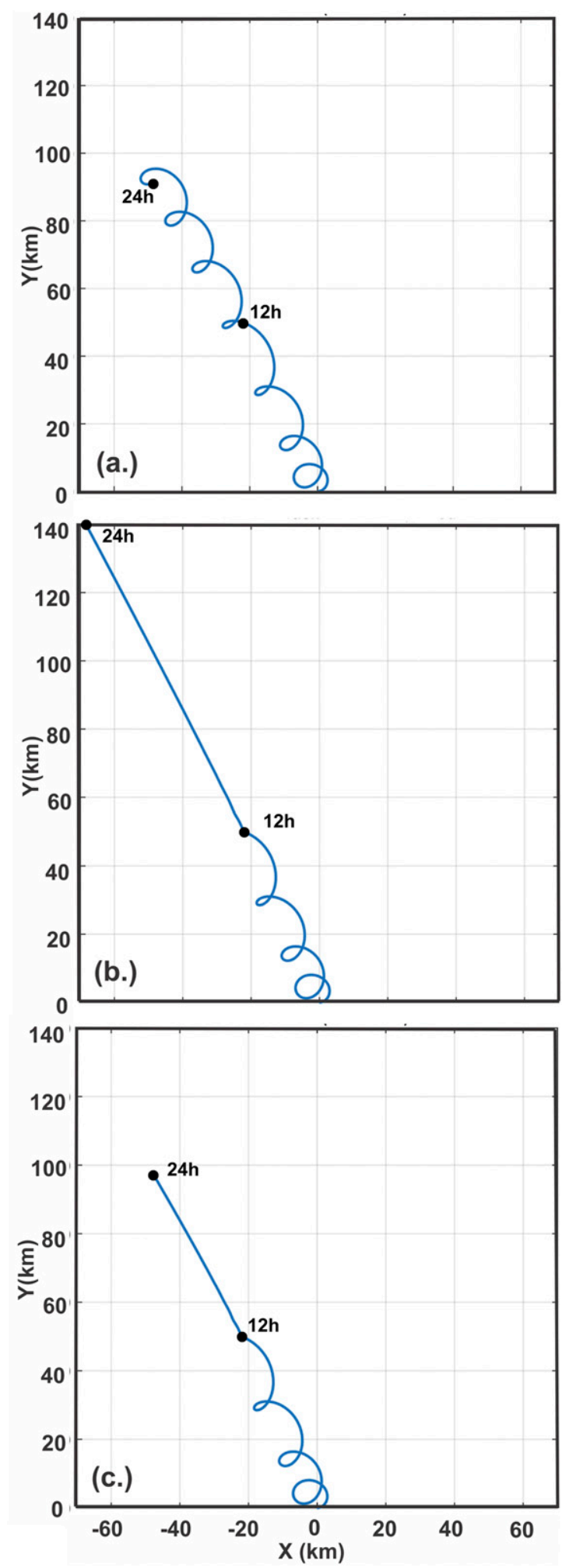

FIG. 10. Simulated $24 \mathrm{~h}$ vortex tracks where (a) the rotating source-sink dipole is active throughout, but the $\beta$ effect artificially turns off at $12 \mathrm{~h}$, (b) the rotating source-sink turns off after $12 \mathrm{~h}$, but the $\beta$ effect remains active throughout, and (c) both the $\beta$ effect and the rotating source-sink turn off after $12 \mathrm{~h}$. relative vorticity increases to zero outward from the small anticyclonic values required by zero circulation at large radius. This configuration suggests the possibility of barotropic instability (e.g., Kossin et al. 2000) even in tropical cyclones that lack an outer wind maximum.

Part II showed that a linear, BND model on a $\beta$ plane, yielded northwestward motion two to 3 times faster than the observed $\beta$ drift for both asymptotically bounded, finitely bounded, and unbounded vortices. Speeds varied substantially, yielding 10-day track lengths that varied from 4400 to $8000 \mathrm{~km}$. In the corresponding nonlinear model, wave-wave interaction dominated the effects of differing mean vortices, which would, in any case, evolve from their initial structure over time. Thus, despite consistency arguments against unbounded profiles, it may not be essential to avoid them as initial conditions in full physics model simulations.

\section{Synoptic-scale Rossby waves}

"Waveguide thinking" confirms and extends Macdonald's (1968) parallels between VRWs and synoptic-scale Rossby waves on a midlatitude $\beta$ plane centered at $45^{\circ}$ latitude. The BND model introduced here is spectral in time and the zonal $(x)$ direction, with specified frequency and zonal wavenumber, and finite difference in the meridional $(y)$ direction. It solves the quasigeostrophic vorticity equation for $\psi$ in a meridionally sheared mean zonal flow and computes wave momentum and geopotential fluxes. The mean-wind profile is a uniform shear, $U(y)=S\left(y-y_{o}\right)$, where $S=0.24 \times 10^{-5}>0$ is the shear, and $y_{o}(500 \mathrm{~km})$ is the transition from easterly to westerly mean flow. The domain extends $\sim 4000 \mathrm{~km}$ from $20^{\circ}$ to $60^{\circ} \mathrm{N}$. The mean flow has weak easterly winds south of $25^{\circ} \mathrm{N}$, and increasing westerly winds that reach $9 \mathrm{~m} \mathrm{~s}^{-1}$ at $60^{\circ} \mathrm{N}$ (Fig. 12a). The waves may be thought of as numerical Green's function solutions excited with $Q$, a $50-\mathrm{km}$-wide sinusoidal forcing at $46^{\circ}$ latitude. The critical latitude and turning latitude are $29^{\circ}$ and $52^{\circ}$, respectively.

The model solves the barotropic, nondivergent vorticity equation expressed in terms of the streamfunction, $\psi(x, y, t)$, such that $u=-\partial \psi / \partial y$ and $v=\partial \psi / \partial x$ :

$$
\left(\frac{\partial}{\partial t}+U \frac{\partial}{\partial x}\right)\left(\frac{\partial^{2} \psi}{\partial x^{2}}+\frac{\partial^{2} \psi}{\partial y^{2}}\right)+\beta \frac{\partial \psi}{\partial x}=Q\left(y_{o}\right) .
$$

In uniform zonal flow, the unforced version of (11) reduces to the familiar dispersion relation for nondivergent plane Rossby waves with constant frequency $\omega$, zonal wavenumber $k$, and meridional wavenumber $\ell$ :

$$
\Omega=\omega-U k=-\frac{\beta k}{k^{2}+\ell^{2}} .
$$

When $\ell$ is large, $\Omega \rightarrow 0$; conversely, when $\lambda$ approaches zero, the dispersion relation is that for a one-dimensional Rossby wave $\Omega \rightarrow-\beta / k=\Omega_{1 \mathrm{D}}$. In the shearing zonal flow, seeking solutions of the form, $\operatorname{Re}\left\{\Psi(y) e^{i(\omega t-k x)}\right\}$, where $\Psi(y)$ is the meridional structure function, produces

$$
(\omega-k U)\left(\frac{d^{2} \Psi}{d y^{2}}-k^{2} \Psi\right)-\beta k \Psi=Q .
$$




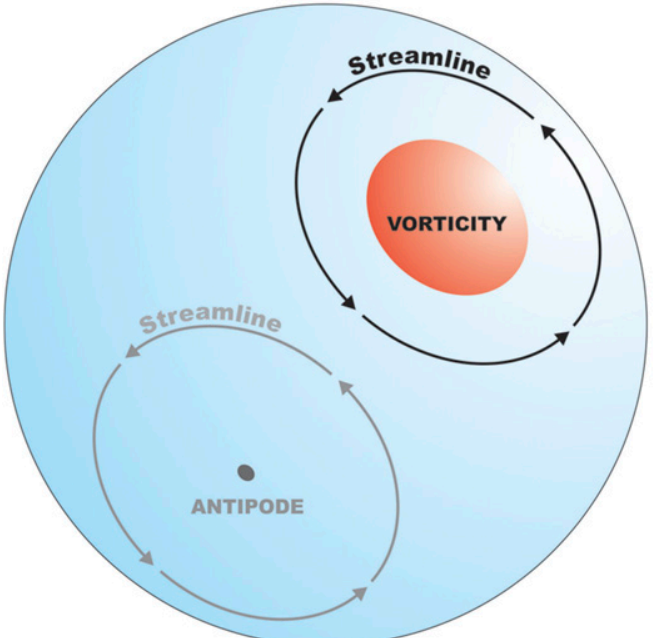

FRONT

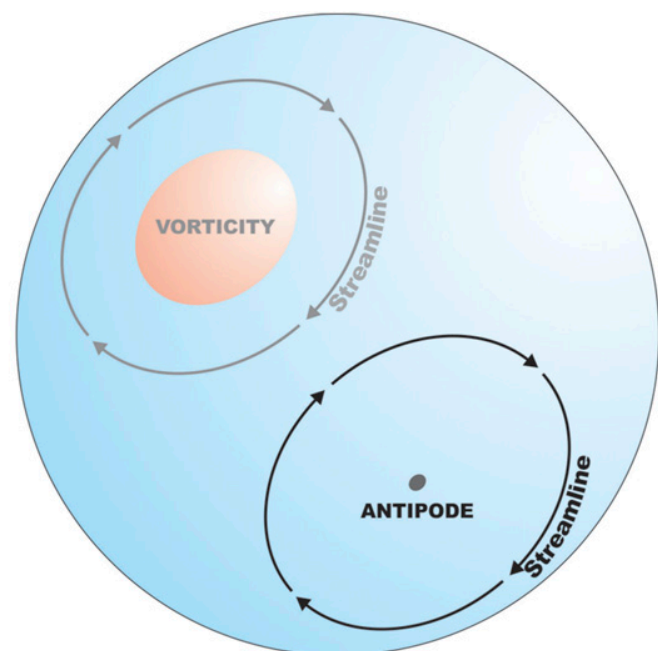

BACK

FIG. 11. An isolated cyclonic vorticity patch on a sphere with no vorticity anywhere else and the apparent contradiction of anticyclonic circulation at the patch's antipode, but with no enclosed vorticity.

As for VRWs, (13) is readily solved for the streamfunction using the method of Lindzen and Kuo (1969). Boundary conditions are $\Psi=0$ at both ends of the domain, far outside the midlatitude waveguide. The solution supports calculation of meridional momentum and geopotential fluxes.

Forcing near the middle of the domain produces a wave train that looks like observed midlatitude cyclones. The commashaped gyres are advected eastward by the mean flow and have curved tails trailing to the southwest. The meridional perturbation flow between the gyres alternates between poleward, westerly flow and equatorward, easterly flow that transport geopotential and momentum. The gyres' domain of active propagation is confined within the boundaries of the Rossby waveguide at $y=900$ and $\sim 3800 \mathrm{~km}$, but evanescent tails of the perturbations extend outside the waveguide, particularly on the poleward side.

As the wave packets propagate away from the forcing latitude, translation frequency $\omega$ and zonal wavenumber $k$ remain constant, but their Doppler-shifted frequencies $\Omega(y)=$ $\omega-k S\left(y-y_{0}\right)$ vary meridionally. $\Omega$ becomes more negative poleward of the forcing latitude and less negative equatorward (Fig. 12a). Thus, equatorward-propagating waves are Doppler shifted to $\Omega=0$ at the critical latitude, where their vorticity becomes filamented. They assume the form of curved, coldfront-like, troughs, and are ultimately absorbed. Dissipation and conservation of wave action as $\Omega \rightarrow 0$ are the absorption mechanisms in the linear context, rather than wave breaking (e.g., McIntyre and Palmer 1983; Homeyer and Bowman 2012).
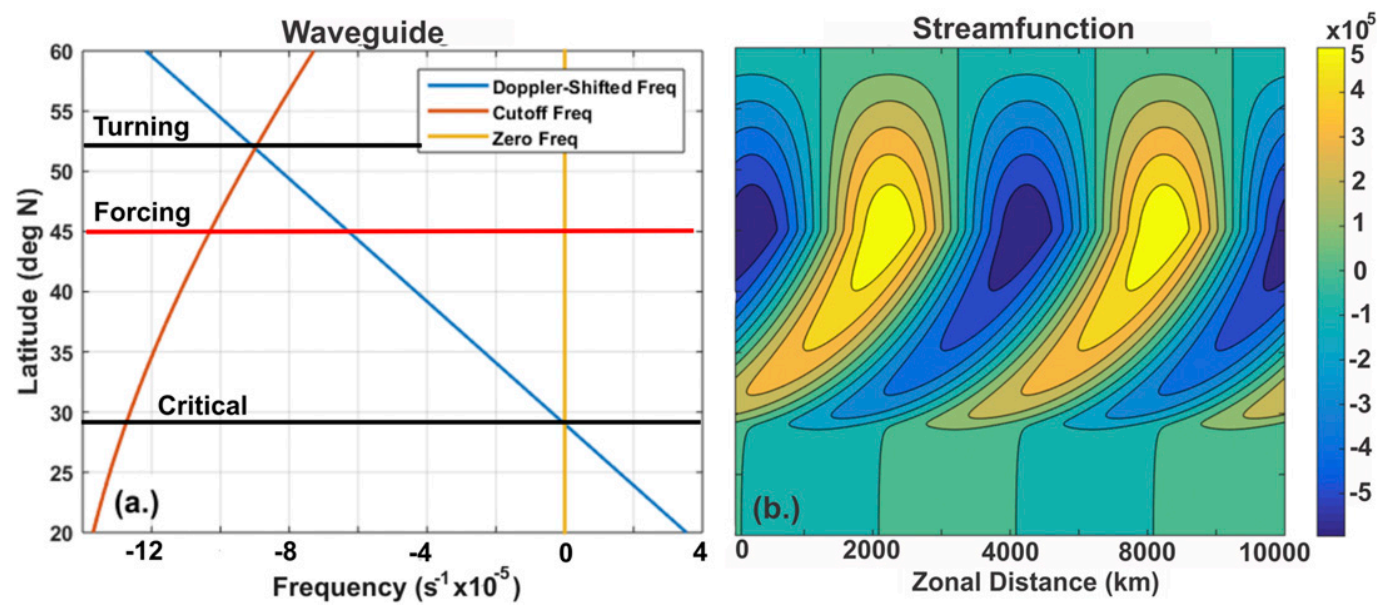

FIG 12. (a) Rossby waveguide bounded on its equatorward side by a critical latitude where the Doppler-shifted frequency $\Omega=0$ and on its poleward side by a turning latitude where $\Omega=-\beta / k$ and (b) streamfunction of a forced, synoptic-scale Rossby wave within this waveguide. 

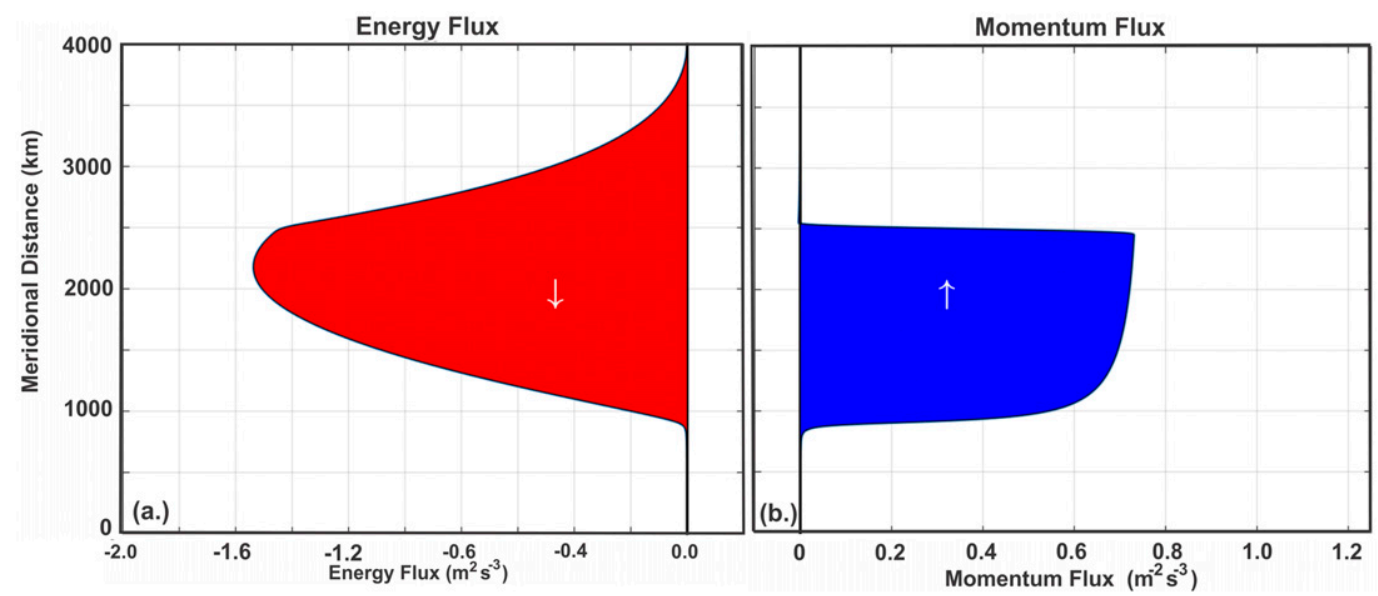

FIG. 13. (a) Meridional wave fluxes of geopotential and momentum, illustrating how forced Rossby waves transport wave energy equatorward and (b) westerly momentum poleward.

The latitude where $\Omega \rightarrow \Omega_{1 \mathrm{D}}=-\beta / k$ is a turning point where initially poleward-propagating waves are Doppler shifted to the highest (most negative) frequency propagating Rossby waves can have. Wave energy reflects from the turning point $\left(\sim 52^{\circ} \mathrm{N}\right)$ and is redirected equatorward where the waves are ultimately Doppler shifted to zero frequency and absorbed as described above, consistent with Karoly and Hoskins (1982), Karoly (1983), and James (1987). On the poleward side of the forcing, between the forcing latitude and the turning point, the waves have a standing-wave structure due to a superposition of the initially poleward-propagating and reflected wave trains. Between the forcing and critical latitudes, the waves propagate equatorward. They are a combination of initially equatorward-propagating and reflected initially poleward-propagating waves (Fig. 12b). They become commashaped gyres that resemble cold fronts trailing from frontal cyclones as they approach the critical latitude. The semicircular half gyres on the poleward side and trailing vorticity filaments suggest the appearance of frontal cyclones on surface analyses.

As expected, the foregoing discussion confirms and extends Macdonald's (1968) analogy between spiral bands and midlatitude cyclones. Momentum and wave energy fluxes (Fig. 13) are also consistent with this analogy. Forced synoptic-scale Rossby waves produce oppositely directed zonal momentum, $\langle v u\rangle$ and geopotential fluxes, $\langle v \phi\rangle$ (e.g., Lorenz and Hartmann 2003). The geopotential flux is negative (equatorward), sustained by both initially equatorward-propagating waves and reflected, initially poleward-propagating, waves. It is largely confined to the equatorward side of the forcing and goes to zero at the critical latitude. The equatorward decrease of the energy flux within this interval (Randel and Held 1991) arises from conservation of wave action, $\langle v \phi\rangle / \Omega(y)$. The zonal momentum flux is poleward and also confined between the forcing and critical latitudes. It is nearly constant with latitude except in the neighborhood of the critical latitude where it is divergent because of absorption. There, it acts to decelerate westerly mean flow (Held and Phillips 1990). It is strongly convergent in a narrow region centered on the forcing latitude, where it acts to accelerate the westerly mean flow (Geisler and Dickinson 1974). Thus, as shown schematically in Fig. 14, the simulated waves act to transport westerly momentum from the subtropics to middle latitudes, just as baroclinically unstable Rossby waves do. The essential difference here is that these are forced, neutral, nondivergent Rossby waves. Nonetheless, these results provide an accessible illustration and extension of "negative viscosity" (Starr 1968) in which energy input to Rossby waves embedded in shearing zonal flow acts to accelerate midlatitude westerly flow, whether the energy comes from baroclinic instability or other sources, such as flow over topography, localized heating, or even tropical cyclones. Shapiro and Ooyama (1990) anticipated that the comma configuration of tropical cyclones is a superposition of planetary Rossby waves ultimately induced by latent heat release in vortex core and the matching between the high Rossby number flow there and the low Rossby number flow in the surroundings. Although the convergence of westerly momentum at latitudes where neutral Rossby waves are forced is a straightforward result of their kinematics, it is not widely appreciated.

\section{Conclusions}

Addressed here are the dynamics of vortex Rossby waves (VRWs) in the context of tropical cyclone (TC) motion and intensity change. The Doppler shift and VRW's relatively slow tangential phase propagation confine them to annular waveguides. Tangential wavenumber-1 VRWs force vortex motion directly and experience the least Doppler shift so that they propagate in radially wider waveguides than wavenumbers $\geq 2$ studied previously.

The simulations address both the time dependent structure of wavenumber-1 VRWs and the meridional structure of a train of synoptic-scale Rossby waves. In both cases the solutions have specified propagation frequency with respect to the ground and tangential, or zonal, wavenumber. The VRWs are 


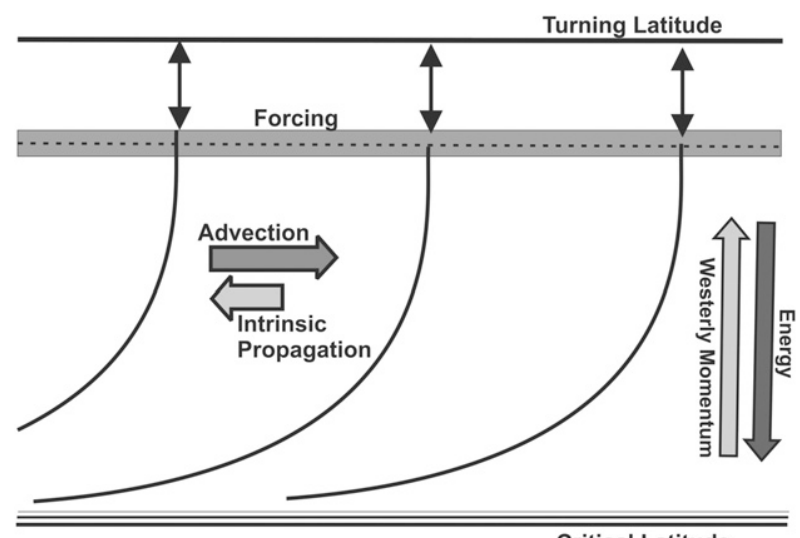

Critical Latitude

FIG. 14. Schematic diagram of synoptic scale Rossby wave propagating in a zonal waveguide, illustrating standing wave structure poleward of the forcing and filamented phase lines equatorward, and the senses of the eddy transports and phase propagation.

simulated on an $f$ plane, forced by an annular, rotating vorticity source-sink pair slightly inward from the radius of maximum wind and propagate upon the radial gradient of mean-vortex relative vorticity. The synoptic-scale waves are forced diabatically or by topography in a narrow band of latitudes on a $\beta$ plane with $f$ and $\beta$ values appropriate to $45^{\circ}$ latitude. Waves that propagate inward, or poleward, from the forcing are Doppler shifted to higher frequency and reflected from a turning radius, or latitude, where their Dopplershifted frequency reaches that of a one-dimensional Rossby wave. They have standing-wave structure. Waves that initially propagate outward, or equatorward, are Doppler shifted to lower frequencies and maintain trailing spiral structure. They evolve into a region of tightly wrapped trailing vorticity filaments as they approach an outer critical radius, or equatorward critical latitude, where their frequencies are Doppler shifted to zero and they are absorbed.

The waves support eddy fluxes of wave energy and momentum. The energy flux diverges both toward higher and lower frequencies from the forcing radius, but the wave train that propagates toward higher frequencies reflects from the turning radius so that the net energy flux is zero inside, or poleward, from the forcing radius. The angular, or westerly, momentum flux is directed from the critical radius to the forcing radius. The momentum flux acts to decelerate the mean flow in the neighborhood of the critical radius and accelerate the mean flow at the forcing radius. For synopticscale waves the equatorward geopotential flux and poleward flux of westerly momentum are consistent with frontal cyclone dynamics. The critical latitude is the locus where the trailing vorticity filaments curve southwestward, reminiscent of the wintertime cold fronts in the southeastern United States.

In the VRW context, combining $\beta$-effect forcing with the rotating vorticity source/sink produces more complex vortex motion-a linear superposition of large-scale northwestward drift and high-frequency orbits that resemble observed trochoidal tracks. The $\beta$-effect translation decays slowly after a shift from a $\beta$ plane to an $f$ plane because of the $\beta$ gyres' wholevortex spatial scale and the slower group velocity at the periphery. When the rotating mass source-sink pair is suppressed on a $\beta$ plane, the track oscillations decay quickly.

Vorticity monopoles are inconsistent with Stokes's theorem on a spherical Earth because the swirling wind would approach infinity as the length of a contour enclosing zero vorticity shrinks to zero length near the antipode. By contrast, bounded vortices, whose circulations approach zero or become identically zero at some finite radius, are consistent with Stokes's theorem. Thus, an annulus of anticyclonic vorticity must surround the inner core of cyclonic vorticity, implying the existence of an outer waveguide, where VRWs propagate faster than the mean flow. Because of a reversed radial vorticity gradient at the vortex periphery, the possibility of barotropic instability exists even without an outer wind maximum.

Future investigations might include seeking unstable barotropic growing modes on a finitely bounded vortex and extending the present calculation to VRWs on a threedimensional baroclinic vortex. More generally, waveguide thinking provides clear insight into vortex Rossby wave dynamics and reinforces the analogy with synoptic-scale Rossby waves. It shows promise as an alternative to the traditional "paper-and-pencil" way of teaching dynamic meteorology by emphasizing numerical solutions of classical problems in terms of waveguides. One may argue that these solutions are richer in terms of phenomenology and closer to the underlying physics.

Acknowledgments. This paper is based upon of the first author's Ph.D. dissertation at FIU. It was supported by NSF Grants AGS-1211172 and AGS-1724198. HEW acknowledges the late J. D. Mahlman, who introduced him to the intricacies of Rossby waves more than a half century ago. Both authors are grateful to Franchesca Espinosa for programming assistance.

Data availability statement. No datasets were generated or analyzed for this study, but Fortran and MATLAB (deprecated) code for the time marching and Lindzen-Kuo algorithms used in the VRW and synoptic-scale problems are available from the second author.

\section{REFERENCES}

Andrews, D. G., and M. E. McIntyre, 1976a: Planetary waves in horizontal and vertical shear: The generalized Eliassen-Palm relation and the mean zonal acceleration. J. Atmos. Sci., 33, 2031-2048, https://doi.org/10.1175/1520-0469(1976)033<2031: PWIHAV $>2.0 . \mathrm{CO} ; 2$.

$\longrightarrow$, and — 1976b: Planetary waves in horizontal and vertical shear: Asymptotic theory for equatorial waves in weak shear. J. Atmos. Sci., 33, 2049-2053, https://doi.org/10.1175/15200469(1976)033<2049:PWIHAV>2.0.CO;2.

Black, M. L., J. F. Gamache, F. D. Marks Jr., C. E. Samsbury, and H. E. Willoughby, 2002: Eastern Pacific Hurricanes Jimena of 1991 and Olivia of 1994: The effect of vertical shear on 
structure and intensity. Mon. Wea. Rev., 130, 2291-2312, https://doi.org/10.1175/1520-0493(2002)130<2291:EPHJOA> 2.0.CO;2.

Boyd, J. P., 1976: The noninteraction of waves with a zonally averaged flow on a spherical Earth and the interrelationships of eddy fluxes of energy, heat and momentum. J. Atmos. Sci., 33, 2285-2291, https://doi.org/10.1175/1520-0469(1976)033<2285: TNOWWT $>2.0 . \mathrm{CO} ; 2$.

Chan, J. C. L., and R. T. Williams, 1987: Analytical and numerical studies of the beta-effect in tropical cyclone motion. Part I: Zero mean flow. J. Atmos. Sci., 44, 1257-1265, https://doi.org/ 10.1175/1520-0469(1987)044<1257:AANSOT>2.0.CO;2.

Chen, Y., and M. K. Yau, 2001: Spiral bands in a simulated hurricane. Part I: Vortex Rossby wave verification. J. Atmos. Sci., 58, 2128-2145, https://doi.org/10.1175/1520-0469(2001)058<2128: SBIASH $>2.0 . \mathrm{CO} ; 2$.

- , and - 2003: Asymmetric structures in a simulated landfalling hurricane. J. Atmos. Sci., 60, 2294-2312, https://doi.org/ 10.1175/1520-0469(2003)060<2294:ASIASL > 2.0.CO;2.

_- G. Brunet, and M. K. Yau, 2003: Spiral bands in a simulated hurricane. Part II: Wave activity diagnostics. J. Atmos. Sci., 60, 1239-1256, https://doi.org/10.1175/1520-0469(2003)60<1239: SBIASH $>2.0 . \mathrm{CO} ; 2$.

Cotto, A., I. Gonzalez III, and H. E. Willoughby, 2015: Synthesis of vortex Rossby waves. Part I: Episodically forced waves in the inner waveguide. J. Atmos. Sci., 72, 3940-3957, https://doi.org/ 10.1175/JAS-D-15-0004.1.

Davis, H. F., 1961: Introduction to Vector Analysis. College Mathematics Series, Allyn and Bacon, Inc., 198 pp.

Gao, C., and P. Zhu, 2016: Vortex Rossby wave propagation in baroclinic, tropical-cyclone-like vortices. Geophys. Res. Lett., 43, 12 578-12 589, https://doi.org/10.1002/2016GL071662.

Geisler, J. E., and R. E. Dickinson, 1974: Numerical study of an interacting Rossby wave and barotropic zonal flow near a critical level. J. Atmos. Sci., 31, 946-955, https://doi.org/ 10.1175/1520-0469(1974)031<0946:NSOAIR > 2.0.CO;2.

Gonzalez, I., III, 2019: Wavenumber-1 vortex Rossby waves propagation in the inner waveguide of a modeled, barotropic nondivergent tropical cyclone. Ph.D. dissertation, Florida International University, 174 pp., https:/digitalcommons.fiu.edu/ etd $/ 4275 /$.

- H. E. Willoughby, and A. Cotto, 2015: Synthesis of vortex Rossby waves. Part II: Vortex motion and waves in the outer waveguide. J. Atmos. Sci., 72, 3958-3974, https://doi.org/ 10.1175/JAS-D-15-0005.1.

Held, I. M., and P. J. Phillips, 1990: A barotropic model of the interaction between the Hadley cell and a Rossby wave. J. Atmos. Sci., 47, 856-869, https://doi.org/10.1175/1520-0469(1990) 047<0856:ABMOTI>2.0.CO;2.

Holland, G. J., 1983: Tropical cyclone motion: Environmental interaction plus a beta effect. J. Atmos. Sci., 40, 328-342, https:// doi.org/10.1175/1520-0469(1983)040<0328:

TCMEIP $>2.0 . C O ; 2$.

Homeyer, C. R., and K. P. Bowman, 2012: Rossby wave breaking and transport between the tropics and extratropics above the subtropical jet. J. Atmos. Sci., 70, 607-626, https://doi.org/ 10.1175/JAS-D-12-0198.1.

James, I. N., 1987: Suppression of baroclinic instability in horizontally sheared flows. J. Atmos. Sci., 44, 3710-3720, https:// doi.org/10.1175/1520-0469(1987)044<3710:SOBIIH>2.0.CO;2.

Karoly, D. J., 1983: Rossby wave propagation in a barotropic atmosphere. Dyn. Atmos. Oceans, 7, 111-125, https://doi.org/ 10.1016/0377-0265(83)90013-1.
— , and B. J. Hoskins, 1982: Three-dimensional propagation of planetary waves. J. Meteor. Soc. Japan, 60, 109-123, https:// doi.org/10.2151/jmsj1965.60.1_109.

Knutson, T. R., and Coauthors, 2010: Tropical cyclones and climate change. Trop. Cyclone Res. Rev., 3, 157-163, https://doi.org/ 10.1016/j.tcrr.2020.01.004.

Kossin, J. P., W. H. Schubert, and M. T. Montgomery, 2000: Unstable interactions between a hurricane's primary eyewall and a secondary ring of enhanced vorticity. J. Atmos. Sci., 57, 3893-3917, https://doi.org/10.1175/1520-0469(2001)058<3893: UIBAHS $>2.0 . \mathrm{CO} ; 2$.

Kuo, H.-C., R. T. Williams, and J.-H. Chen, 1999: A possible mechanism for the eye rotation of Typhoon Herb. J. Atmos. Sci., 56, 1659-1673, https://doi.org/10.1175/1520-0469(1999) 056<1659:APMFTE $>2.0 . \mathrm{CO} ; 2$.

Lawrence, M. B., and B. M. Mayfield, 1977: Satellite observations of trochoidal motion during Hurricane Belle 1976. Mon. Wea. Rev., 105, 1458-1461, https://doi.org/10.1175/1520-0493(1977) 105<1458:SOOTMD $>2.0$. CO;2.

Lindzen, R. S., and H. L. Kuo, 1969: A reliable method for the numerical integration of a large class of ordinary and partial differential equations. Mon. Wea. Rev., 97, 732-734, https://doi.org/10.1175/1520-0493(1969)097<0732:ARMFTN> 2.3.CO;2.

Lorenz, D. J., and D. L. Hartmann, 2003: Eddy-zonal flow feedback in the Northern Hemisphere winter. J. Atmos. Sci., 16, 1212-1227, https://doi.org/10.1175/1520-0442(2003)16<1212: EFFITN $>2.0 . \mathrm{CO} ; 2$.

Möller, J. D., and L. J. Shapiro, 2002: Balanced contributions to the intensification of Hurricane Opal as diagnosed from a GFDL model forecast. Mon. Wea. Rev., 130, 1866-1881, https://doi.org/10.1175/1520-0493(2002)130<1866:BCTTIO > 2.0.CO;2.

Macdonald, N. J., 1968: The evidence for the existence of Rossbytype waves in the hurricane vortex. Tellus, 20,138-150, https:// doi.org/10.3402/tellusa.v20i1.9993.

McIntyre, M. E., and T. N. Palmer, 1983: Breaking planetary waves in the stratosphere. Nature, 305, 593-600, https://doi.org/ 10.1038/305593a0.

Montgomery, M. T., and R. J. Kallenbach, 1997: A theory for vortex Rossby-waves and its application to spiral bands and intensity changes in hurricanes. Quart. J. Roy. Meteor. Soc, 123, 435-465, https://doi.org/10.1002/qj.49712353810.

Muramatsu, T., 1986: The structure of polygonal eye of a typhoon. J. Meteor. Soc. Japan, 64, 913-921, https://doi.org/10.2151/ jmsj1965.64.6_913.

Nolan, D. S., and M. T. Montgomery, 2000: The algebraic growth of wavenumber one disturbances in hurricane-like vortices. J. Atmos. Sci., 57, 3514-3538, https://doi.org/10.1175/15200469(2000)057<3514:TAGOWO>2.0.CO;2.

,-- , and L. D. Grasso, 2001: The wavenumber-one instability and trochoidal motion of hurricane-like vortices. J. Atmos. Sci., 58, 3243-3270, https://doi.org/10.1175/15200469(2001)058<3243:TWOIAT $>2.0$.CO;2.

Randel, W. J., and I. M. Held, 1991: Phase speed spectra of transient eddy fluxes and critical layer absorption. J. Atmos. Sci., 48, 688-697, https://doi.org/10.1175/1520-0469(1991)048<0688: PSSOTE $>2.0 . \mathrm{CO} ; 2$.

Reasor, P. D., M. T. Montgomery, F. D. Marks Jr., and J. F. Gamache, 2000: Low-wavenumber structure and evolution of the hurricane inner core observed by airborne dual-Doppler radar. Mon. Wea. Rev., 128, 1653-1680, https://doi.org/10.1175/ 1520-0493(2000)128<1653:LWSAEO>2.0.CO;2. 
Riehl, H., 1963: Some relations between wind and thermal structure of steady-state hurricanes. J. Atmos. Sci., 20, 276-287, https://doi.org/10.1175/1520-0469(1963)020<0276:SRBWAT> 2.0.CO;2.

Shapiro, L. J., and K. V. Ooyama, 1990: Barotropic vortex evolution on a beta plane. J. Atmos. Sci., 47, 170-187, https://doi.org/ 10.1175/1520-0469(1990)047<0170:BVEOAB > 2.0.CO;2.

Starr, V. P., 1968: Physics of Negative Viscosity Phenomenon. McGraw-Hill, Inc., 256 pp.

Tsai, Y.-M., H.-C. Kuo, and W. S. Shubert, 2010: Filamentation time diagnosis of thinning troughs and cutoff lows. Mon. Wea. Rev., 138, 2327-2335, https://doi.org/10.1175/2010MWR3102.1.
Wang, Y., 2002: Vortex Rossby waves in a numerically simulated tropical cyclone. Part II: The roles in tropical cyclone structure and intensity changes. J. Atmos. Sci., 59, 1239-1262, https://doi.org/ 10.1175/1520-0469(2002)059<1239:VRWIAN >2.0.CO;2.

Willoughby, H. E., 1992: Linear motion of a shallow-water barotropic vortex as an initial-value problem. J. Atmos. Sci., 49, 2015-2031, https://doi.org/10.1175/1520-0469(1992)049<2015: $\mathrm{LMOASW}>2.0 . \mathrm{CO} ; 2$.

Wood, V. T., and L. W. White, 2010: A new parametric model of vortex tangential-wind profiles: Development, testing, and verification. J. Atmos. Sci., 68, 990-1006, https://doi.org/ 10.1175/2011JAS3588.1. 